\title{
Diagnosis of Alzheimer's Diseases from MRI Images using Image Processing and Machine Learning Approach
}

\author{
Vandana B.S. \\ Dept of CS\&E \\ Vivekananda College of Engineering and \\ Technology \\ Puttur, Dakshina Kannada, Karnataka, India
}

\author{
Sathyavathi R. Alva \\ Department of Pathology \\ KVG Medical College \\ Sullia, RGUHS, India
}

\begin{abstract}
Alzheimer disease is an incurable, progressive neurological brain disorder. Earlier detection of Alzheimer's disease can help with proper treatment and prevent brain tissue damage. In this work we proposed two methods. Radiological feature extraction using image processing and machine learning from MRI images and Analysis of Alzheimer'sdiseases state by using deep learning approach. In the first phase, the algorithm first normalizes and removes skull from the MRI images. Modified K-Means algorithm is used to partition the image into white matter (WM), grey matter (GM) and black holes $(\mathrm{BH})$. The relevant diagnostic features are extracted from the segmented image component. The classifier is trained by the training data to predict the test data. The features are defined to construct classification model by using Support Vector Machine. In the above techniques, the work was carried out with specific features. Unlike this, deep learning method studies profound features from lower level to higher level without human intervention. Here, database contains total of 1000 images which are resized into $350 \times 350$ without loss of information. Deep Learning demands large number of images and its strength was increased as per requirement by augmentation technique. In the first phase of the method takes 1000 images of different features are selected to train SVM classifier and the accuracy obtained is $92 \%$. In the second Phase success rate is $85.6 \%$, and contribution of this work is classification of images into categories such as Alzheimer (AD)and normal. First phase of workemphasized program specific applications to extract features.In the second phase the CNN multiple layers which are studied from lower level to the higher-level image characteristics.
\end{abstract}

\section{Keywords}

Alzheimer, Classification, Deep-Learning, MRI images, Segmentation

\section{INTRODUCTION}

Alzheimer's disease is the most prevalent type of mental illness. It happens when plaques containing beta amyloid form in the brain. As it intensifies, it becomes difficult for patient to recall situations and to recognize some people $[1,2]$. Around $60 \%$ of people of all ages have Alzheimer's. An approximate of 6 million Americans of age 60 and older are living with dementia in 2020[3]. Laboratory test and brain imaging tests provide us important diagnostic feature of Alzheimer's dementia disorder. The CT scan is used to characterize the patterns of cortical atrophy but MRI is more sensible and better able to differentiate other causes of dementia $[4,5]$. The main diagnostic feature in MRI of Alzheimer disease is the estimation of volume change in brain characteristic locations and such volume loss will not manifest early [6]. Mesial temporal lobe atrophy specially in the hippocampus and entorhinal cortex. Volumetric analysis of hippocampus has to be determined to identify the tissue damage in patients with temporal lobe epilepsy. Correlations between the volumes of these structures should be analyzed. The brain cells degenerate and die forming a hallow space in the grey portion of the brain. Brain volume analysis estimated with segmentation, signifies that patient with Alzheimer disease have higher rates of volume loss around twice normal per year. This is even more in the hippocampi, exhibiting three times the volume loss per year [7]. The automation research work was initiated few years ago $[8,9,10]$. Researchers have developed Computer Aided Diagnosis (CAD) systems influenced to obtain maximum images for the study in order to automatically diagnose $\mathrm{AD}[11,12,13]$.

In this view several methods for a detection of Alzheimer's disease using functional and structural images have been proposed $[14,15]$. All these approaches composed of three phases. Pre-processing, Segmentation, Feature extraction and classification $[16,17,18]$.

\subsection{Objectives}

The proposed work includes designing a method, which perform accurate segmentation to define all the features of Alzheimer MRI image and classification of these features to define the disease state. A tool that allows analysis and generates confidence in results augments the role of the Radiologist. The project activity will include the following objectives.

- Perfect segmentation of all features presented in the radiological image.

- $\quad$ Detecting the disease state.

\section{DATASETS}

Alzheimer' Disease affected MRI plays pivotal role in this research work [19]. We use standard dataset collected from the Alzheimer's Disease Neuroimaging Initiative (ADNI). ADNI is a pool of MRI data which promotes the researchers to work on the progression of Alzheimer's disease (AD) [20]. The collected dataset of MR brain image is in NIFTI format (.nii). Radiologist derive diagnosis observations from these images. These findings cater the need of a radiologist through simplifying diagnosis process with accurate declaration of disease. 


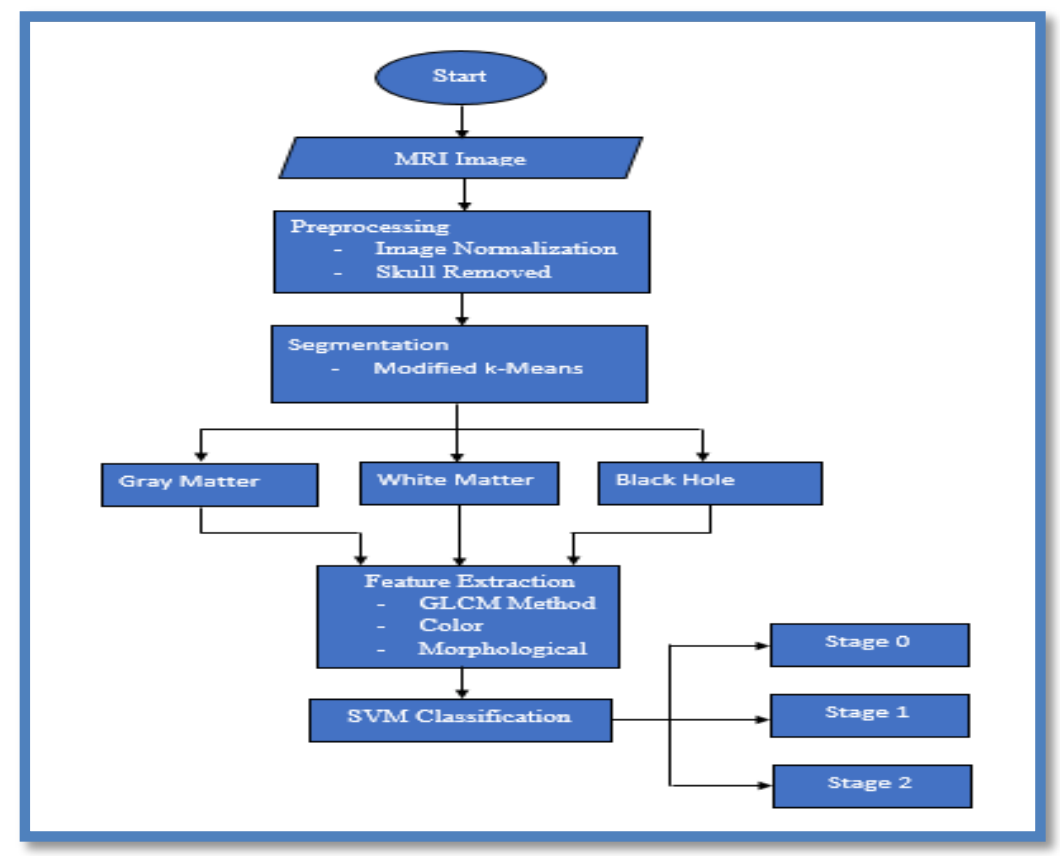

Figure 1: Framework of Alzheimer's disease Detection

\section{METHODOLOGY}

In this direction, basic features of Alzheimer images of MRI are studied and developed the algorithm for automated diagnosis. Detection of diagnostic factors from the radiological image of Alzheimer's disease is challenging due to the complex architecture of MRI sample. Some methods proposed in the past for the detection of abnormalities in MRI imagery. These approaches taken into consideration. The algorithms are developed to extract different properties related to the volume loss, presence of parietal atrophy. Machine learning methods emphasized on program specific application to extract specific features for defining disease state. In the above technique, work was carried out with specific features. Unlike this, deep learning method studies profound features from lower level to higher level without human intervention. Deep learning method with the concepts of image processing and machine learning techniques are adopted for Alzheimer's disease detection. Though the previous work was efficient, but still latest technology can improve the research work. The frame work of this proposed method 1 is depicted in following figure1.

\subsection{Method1: Radiological feature extraction using image processing and machine learning approach from MRI images}

\subsubsection{Pre-processing}

This process includes normalization and noise removal from the MRI Images. The noise that is formed in the images may be due to the environmental factors. The skull being of no use to the detection of the disease, it is eliminated through morphological operation such as opening and closing.

\subsubsection{Image Segmentation}

Segmentation is a process where the image is divided into non-overlapping regions of similar characteristics. The normalized image is given to the segmentation module. $\mathrm{K}$ Means Clustering algorithm is a classical method for grouping image objects based on color. Input parameter $\mathrm{k}$ is given in advance; accordingly, it will make k-groups out of $\mathrm{n}$ objects. This method partitions the image into three classes, namely white matter (WM), grey matter (GM) and black holes (BH). This step makes it easy to extract volumetric features from every image component.

\subsubsection{Feature Extraction and Classification}

Grey Level Co-occurrence Matrix (GLCM) Features are compute from the segmented image components. Grey-level co-occurrence matrix (GLCM) is a statistical method used for examining texture while keeping in view the spatial resolution of pixels. GLCM calculates occurrences of pixel pairs with specified values that are in a relationship with each other to determine texture of image. These occurrences are then used to compute statistical information from GLCM matrix. Cooccurrence matrix has been used to extract contrast, correlation, homogeneity, and entropy.

The selected is SVM, are trained to classify data into normal (1), mild stage (2) and Severe Stage (3) class using supervised learning.

\subsubsection{Result}

Total 1000 MRI images which includes both AD as well as normal are used for experiment. Chosen dataset is grouped as training set with $60 \%$ images and test sets with remaining images for validation. 


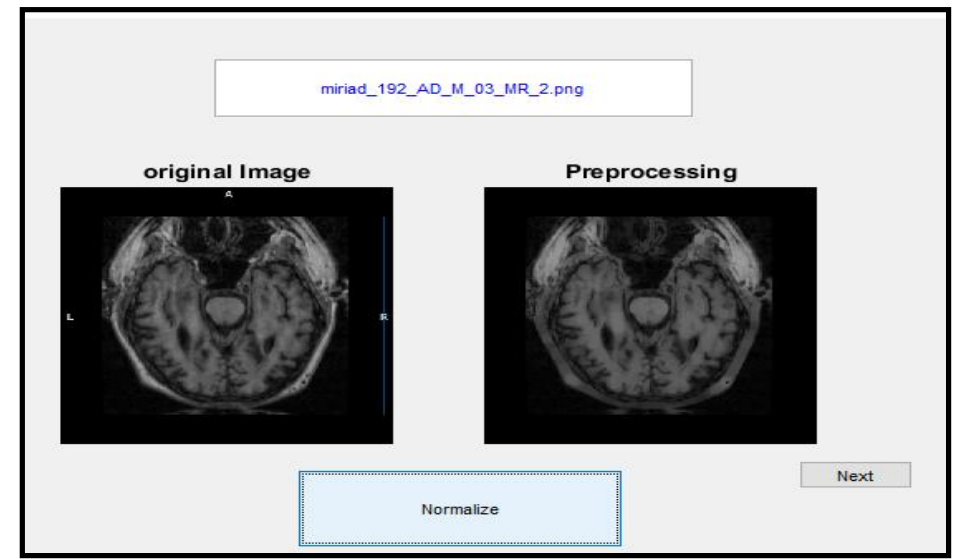

Figure 2(a): Result of Pre-processing

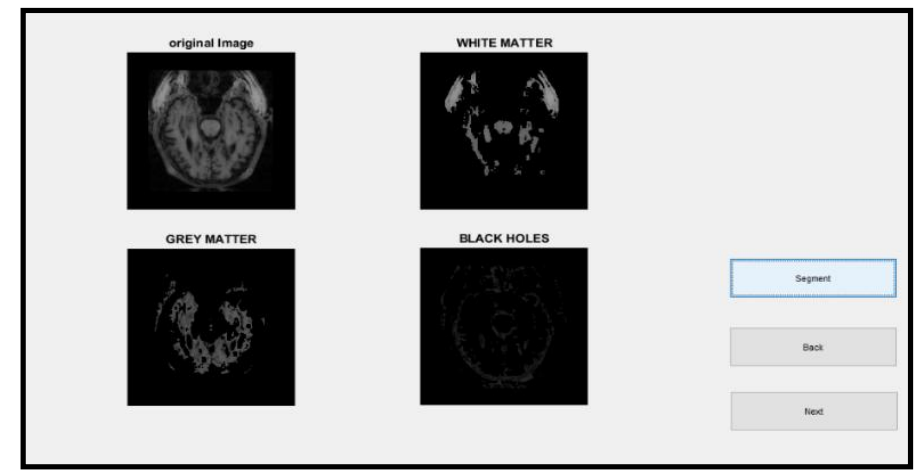

Figure 2(b) Result of segmentation into white matter, grey matter, black holes component

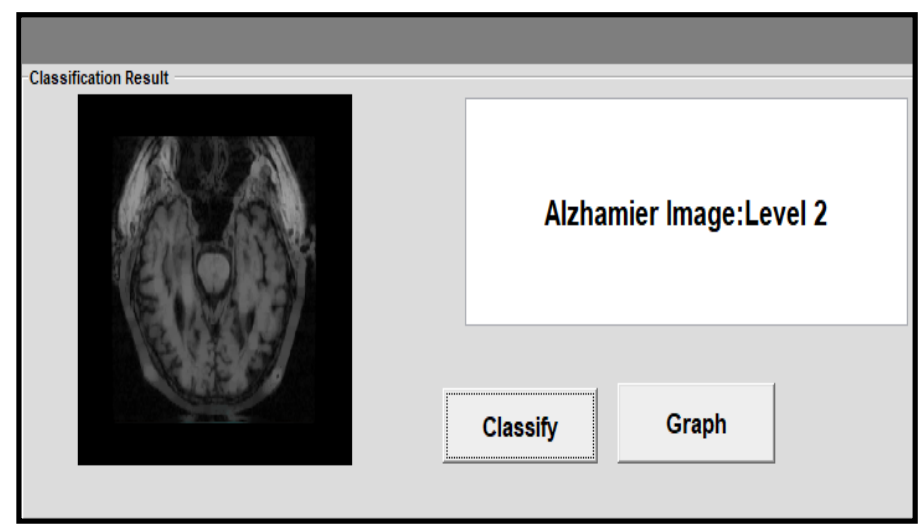

Figure 3: Result of automated frame work

The Pre-processing algorithm includes histogram equalization would apply only on images and used for improving the quality of the images. Subsequently we removed Skull from the input image to enhance the efficiency of segmentation algorithm and the result is shown in the figure 2(a). Modified version of K-Means algorithm maximizes the ability to predict the pixel of an image that assigns to the correct cluster. The segmented image component is demonstrated in the figure 2(b).

\subsubsection{Classification Accuracy}

This proposed work has good result with classification accuracy of $92 \%$. Confusion metrics and Evaluation metrics are given in Table 1. Dataset sample of 1000 images - $\mathbf{2 0 0}$ normal, $\mathbf{8 0 0}$ diseases affected, the resulting confusion matrix is given below:In the proposed approach, GLCM, grey matter proportion and white matter volume to cerebrospinal volume ratio along with clinical features are used for classification. The resultsindicate that using clinical features and the computed texture-based features can boost classification accuracy significantly. Multiclass classification for AD, NC and Mild Stage is also addressed although it is very challenging due to similarity between $\mathrm{AD}$ and Mild Stage subjects. The proposed method achieves significant accuracy for multi-class classification and result is depicted in figure 3 . 
Table 1: Classification Result of method 1.

\begin{tabular}{|l|l|l|}
\hline Class & C1 & C2 \\
\hline C1(Normal) & 160 & 40 \\
\hline C2(AD) & 40 & 760 \\
\hline Accuracy & \multicolumn{2}{|c|}{$92 \%$} \\
\hline
\end{tabular}

\subsection{Method 2: Analysis of Alzheimer's diseases state by using Deep Learning Approach}

In deep learning, a convolutional neural network is a class of deep neural networks, most commonly applied to analyzing visual imagery. Here our task is image classification, which is the process of taking an input and provides the Alzheimer's disease state. Technically, deep learning CNN models to train and test, each input image will pass it through a series of convolution layers with filters, Pooling, fully connected layers (FC) and apply SoftMax function to classify an object with probabilistic values between 0 and 1 .

\subsubsection{Dataset}

The dataset are MRI images of Alzheimer's diseases. All these images are same size and resolution. This dataset contains 1000 images which are resized into $350 * 350$ without loss of information. This dataset includes normal and Alzheimer's diseases (AD) affected images.

Proposed method includes the following steps

$>$ Image augmentation.

$>$ Data preparation.

$>$ Feature extraction and image classification

\subsubsection{Image augmentation}

Deep learning needs large amount of training data to achieve good performance. This method usually required to boost the performance of deep networks. Image augmentation artificially creates training images. 1000 image dataset boosted up into 2000 images.

\subsubsection{Data preparation}

There are three types of images in the single folder. Images are labelled as Normal. 0,AD. 1 .Here 0 and 1 are the class label. This group of images are randomly grouped as training datasets and test datasets.

\subsubsection{Feature extraction and image classification}

Convolution neural network learning on multiple layers. Every layer has convolution and pooling method. These layers are used to learn spatial characteristics of MRI image. In Convolution layer image is convolved with chosen filters, taking dot product with portions of image pixels. The ReLu activation function is used here.

Pooling layer reduces spatial size of feature map received from convolution layer which will minimize the computation complexity. In this procedure max pooling technique is used to read every feature map distinctly. After feature extraction we need to classify the data into various classes. This can be done using fully connected neural Network. The steps are provided in algorithm 1 .

Algorithm 1: Image Classification Using CNN architecture Input: Array of pixel values [height, width, channel] Feature Extraction

1 Apply Convolution neural network to get feature map

1.1 Convolution (ReLu)

1.1.1 Select the kernel which has the same depth as input array and its size is $5 \times 5$.

1.1.2 Perform convolution operation to get image features 1.2 Pooling (Max Pooling)

1.2.1 Reduce the spatial size of feature map using dimensionality reduction process and extract dominant feature and its size is $2 \times 2$

2 Repeat the above process till $4^{\text {th }}$ layer by varying channel size from 16,32,64, and 128 to get low level feature from the image.

\section{Classification}

3 During each iteration of the training phase, flat output is given to a feed-forward neural network with back propagation.

4 Trained model is used to classify by differentiating dominant features in the images and classify them with using the SoftMax Classification technique.

\subsubsection{Result interpretation}

Apply CNN to the MRI image of volume $350 \times 350 \times 3$. When the convolution procedure is completed the depth of input is amplified by the number of filters used. When the Max pooling is applied depth remains the same and representation size is compressed. Next, add a dense layer to CNN model to achieve classification. Convolution layer result will be further flattened and provided to SoftMax function. The particulars of the CNN architecture are illustrated in Table 2 and Table 3. All experiments were carried out on a computer (Intel Core (TM) $2.4 \mathrm{GHz}$ processor with $8 \mathrm{~GB}$ of RAM). The software implementation was performed using Python. 
Table 2: CNN parameter

\begin{tabular}{|c|c|c|c|c|c|}
\hline & CHANNEL & VOLUME & $\begin{array}{l}\text { FILTER } \\
\text { SIZE }\end{array}$ & STRIDE & PADDING \\
\hline Input Image & - & $350 \times 350 \times 3$ & - & - & Same \\
\hline convolution layer 1 & 16 & $346 \times 346 \times 16$ & $5 \times 5$ & 1 & - \\
\hline max pooling 1 & - & $173 \times 173 \times 16$ & $2 \times 2$ & 2 & same \\
\hline convolution layer 2 & 32 & $169 \times 169 \times 32$ & $5 \times 5$ & 1 & none \\
\hline max pooling 2 & - & $84 \times 84 \times 32$ & $2 \times 2$ & 2 & - \\
\hline convolution layer 3 & 64 & $80 \times 80 \times 64$ & $5 \times 5$ & 1 & none \\
\hline max pooling 3 & - & $40 \times 40 \times 64$ & $2 \times 2$ & 2 & - \\
\hline convolution layer 4 & 128 & $36 \times 36 \times 128$ & $5 \times 5$ & 1 & 2 \\
\hline max pooling 4 & & $18 \times 18 \times 128$ & $2 \times 2$ & & 2 \\
\hline
\end{tabular}

Table 3: CNN classification parameter

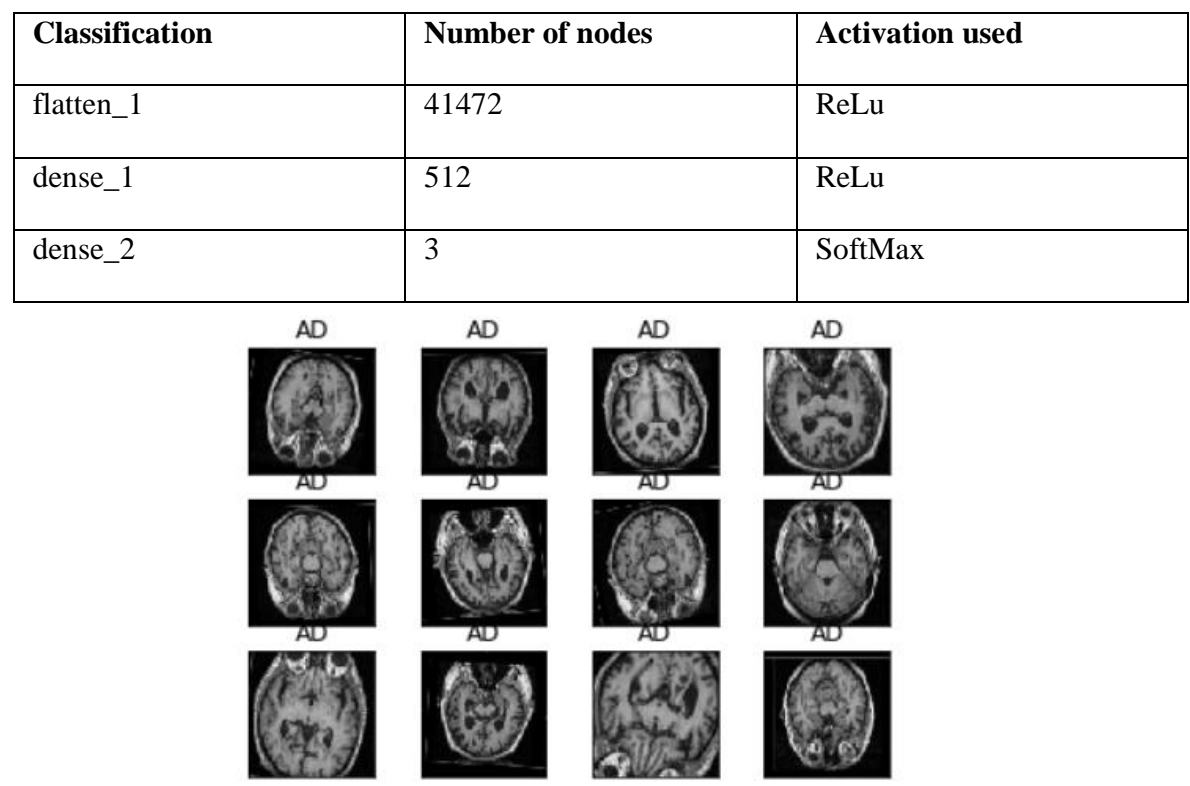

Figure 4: Result of automated tool which classifies AD image as AD

\subsection{Classification Accuracy}

This proposed work has tremendous result with classification accuracy of $85.6 \%$. and result is shown in figure 4 . Confusion metrics and Evaluation metrics are given in Table 4. Dataset sample of 2000 images - 400 normal, 1600 disease affected, the resulting confusion matrix is given below:

Table 4: Classification Accuracy of method 2

\begin{tabular}{|c|c|c|}
\hline class & C1 & C2 \\
\hline $\mathrm{C} 1$ (Normal) & 312 & 88 \\
\hline $\mathrm{C} 2(\mathrm{AD})$ & 200 & 1400 \\
\hline Accuracy & & $85.6 \%$ \\
\hline
\end{tabular}




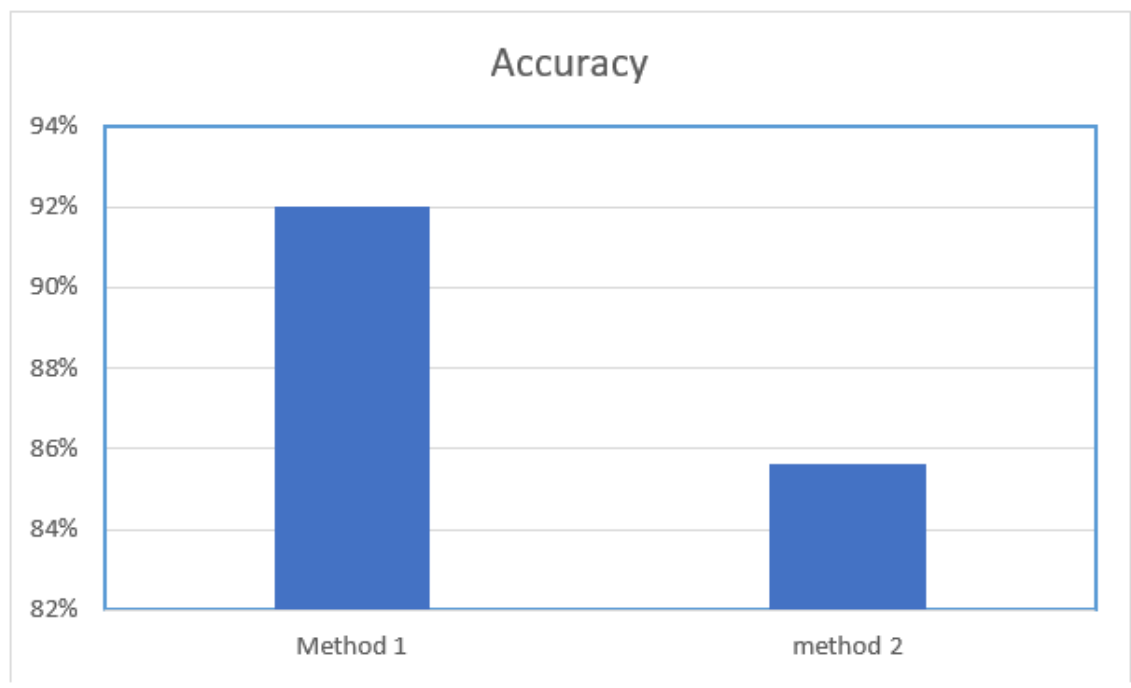

Figure 5: Shows accuracy plot of two methods

Previous method-based segmentation result was good because doctors who considers same features in the diagnosis process. In the first level result indicates $92 \%$ accuracy due to its program specific features which are taken into account. In Deep Learning Technique $85.6 \%$ accuracy was attained. Existing images are strengthened as per requirement for image processing. Here, all the features are studied on its own without specific feeding. Accuracy of both the method are illustrated in figure 5 .

\section{CONCLUSIONS}

The first method is based on domain specific algorithm where classification result is based on specific feature that represents the subjective knowledge of doctors. Existing images are strengthened as per requirement for image processing. Here, all the features are studied on its own without specific feeding.

Despite enormous medical specialist's available success of diagnosis is a nightmare. Without technology medical field is incompatible with the speed of disease outbreak. Thus, constant collaboration of Radiologists along with Computer Vision researchers are indispensable for the success. The dovetailing pursuit was attempted in the computer aided diagnosis for Alzheimer's disease.

\section{REFERENCES}

[1] Yaakov Stern, Barry Gurland, Thomas K Tatemichi, Ming Xin Tang, David Wilder, and Richard Mayeux. Incidence of education and occupation on the incidence of Alzheimer's disease. Jama, 271(13):1004-1010, 1994.

[2] Jeffrey L Cummings and Greg Cole. Alzheimer disease. Jama,287(18):2335-2338, 2002.

[3] 2020 Alzheimer's disease facts and figures. Alzheimer's \& Dementia,16(3):391-460.

[4] Rudy J Castellani, Raj K Rolston, and Mark A Smith. Alzheimer disease. Disease-a-month: DM, 56(9):484, 2010.

[5] Alberto Serrano-Pozo, Matthew $P$ Frosch, Eliezer Masliah, and Bradley $\mathrm{T}$ Hyman. Neuropathological alterations in Alzheimer disease. Cold Spring Harbor perspectives in medicine, 1(1): a006189, 2011.
[6] Brenda L Plassman, Kenneth M Langa, Gwenith G Fisher, Steven G Heeringa, David R Weir, Mary Beth Ofstedal, James R Burke, Michael D Hurd, Guy G Potter, Willard L Rodgers, et al. Prevalence of cognitive impairment without dementia in the united states. Annals of internal medicine, 148(6):427-434, 2008.

[7] Jennifer L Whitwell, Cli_ord R Jack Jr, Scott A Przybelski, Joseph E Parisi, Matthew L Senjem, Bradley F Boeve, David S Knopman, Ronald C Petersen, Dennis W Dickson, and Keith A Josephs. Temporoparietal atrophy: a marker of ad pathology independent of clinical diagnosis. Neurobiology of aging, 32(9):1531\{1541, 2011.

[8] Carolyn Cidis Meltzer, Jeffrey P Leal, Helen S Mayberg, Henry N Wagner Jr, and J James Frost. Correction of pet data for partial volume effects in human cerebral cortex by $\mathrm{mr}$ imaging. Journal of computer assisted tomography, 14(4):561-570, 1990.

[9] GB Frisoni, C Testa, A Zorzan, F Sabattoli, A Beltramello, H Soininen, and MP Laakso. Detection of grey matter loss in mild Alzheimer's disease with voxelbased morphometry. Journal of Neurology, Neurosurgery \& Psychiatry, 73(6):657-664, 2002.

[10] Satoshi Minoshima, Kirk A Frey, Norman L Foster, and David E Kuhl. Preserved pontine glucose metabolism in Alzheimer disease: a reference region for functional brain image (pet) analysis. Journal of computer assisted tomography, 19(4):541-547, 1995.

[11] Javier Ramirez, JM Gorriz, Diego Salas-Gonzalez, A Romero, Miriam Lopez, Ignacio Alvarez, and Manuel Gomez Rio. Computer-aided diagnosis of Alzheimer's type dementia combining support vector machines and discriminant set of features. Information Sciences, 237:59-72, 2013.

[12] MM Lopez, J Ramirez, JM Gorriz, I Alvarez, D SalasGonzalez, F Segovia,and R Chaves. Svm-based cad system for early detection of the Alzheimer's disease using kernel pca and lda. Neuroscience Letters,64(3):233-238,2009.

[13] Laila Khedher, Ignacio A Illan, Juan M Gorriz, Javier Ramirez, AbdelbassetBrahim, and Anke Meyer-Baese. 
Independent component analysis-support vector machine-based computer-aided diagnosis system for Alzheimer's with visual support. International journal of neural systems, 27(03):1650050, 2017.

[14] Philip Scheltens, Nick Fox, Frederik Barkhof, and Charles De Carli. Structural magnetic resonance imaging in the practical assessment of dementia:beyond exclusion. The Lancet Neurology, 1(1):13-21, 2002.

[15] Yu-Dong Zhang, Shuihua Wang, and Zhengchao Dong. Classi_cation of alzheimer disease based on structural magnetic resonance imaging by kernel support vector machine decision tree. Progress In Electromagnetics Research, 144:171-184, 2014.

[16] Nilanjan Dey, Amira S Ashour, Sayan Chakraborty, Sourav Samanta, Dimitra Sifaki-Pistolla, Ahmed S Ashour, Dac-Nhuong Le, and Gia NhuNguyen.Healthy and unhealthy rat hippocampus cells classi_cation: A neural based automated system for alzheimer disease classification. Journal of Advanced Microscopy Research, 11(1):1\{10, 2016.
[17] Weiming Lin, Tong Tong, Qinquan Gao, Di Guo, Xiaofeng Du, YongguiYang, Gang Guo, Min Xiao, Min $\mathrm{Du}$, Xiaobo Qu, et al. Convolutional neural networksbased mri.

[18] Ruoxuan Cui, Manhua Liu, Alzheimer's Disease Neuroimaging Initiative,et al.Rnn-based longitudinal analysis for diagnosis of alzheimersdisease.Computerized Medical Imaging and Graphics, 73:1\{10, 2019.

[19] Keith A Johnson, Nick C Fox, Reisa A Sperling, and William E Klunk.Brain imaging in alzheimer disease. Cold Spring Harbor perspectives in medicine, 2(4):a006213, 2012.

[20] Ricardo Guerrero, RobinWolz, AWRao, Daniel Rueckert, Alzheimer's Disease Neuroimaging Initiative (ADNI, et al. Manifold population modelling as a neuroimaging biomarker: application to adni and adni-go. NeuroIm-age, 94:275-286, 2014. 